\title{
Parallel Model Checking for the Alternation Free $\mu$-Calculus
}

\author{
Benedikt Bollig, Martin Leucker, and Michael Weber \\ Lehrstuhl für Informatik II, RWTH Aachen, Germany \\ \{bollig, leucker, michaelw\}@i2.informatik.rwth-aachen.de
}

\begin{abstract}
In this paper, we describe the design and implementation of a parallel model checking algorithm for the alternation free fragment of the $\mu$-calculus. It exploits a characterisation of the model checking problem for this fragment in terms of two-person games. Our algorithm determines the corresponding winner in parallel. It is designed to run on a network of workstations. An implementation within the verification tool TRUTH shows promising results.
\end{abstract}

\section{Introduction}

Model checking is becoming more and more popular for the verification of complex hardware and software systems. These systems are usually given by a formal description which can be transformed into a transition system. A desired property of the system, on the other hand, is usually specified as a formula of a temporal logic. A model checking algorithm answers the question whether the (transition) system satisfies this property. Numerous case studies have shown that this approach improves the early detection of errors 3 .

Despite the developments in the last years, the so-called state space explosion still limits its application. While partial order reduction 20 or symbolic model checking 17 reduce the state space by orders of magnitude, typical verification tasks still last days on a single workstation or are even (practically) undecidable due to memory restrictions (see for example 7 ).

On the other hand, cheap yet powerful parallel computers can be constructed of Networks Of Workstations (NOWs). From the outside, a NOW appears as one single parallel computer with high computing power and, even more important, huge amounts of memory. This enables parallel programs to utilise the accumulated resources of a NOW to solve large problems. Hence, it is a fundamental goal to find parallel model checking algorithms which then may be combined with well-known techniques to avoid the state space explosion gaining even more speedup and further reducing memory requirements.

A famous logic for expressing specifications is Kozen's $\mu$-calculus II, a temporal logic offering boolean combination of formulae and, especially, labelled next-state, minimal, and maximal fixpoint quantifiers. For practical applications, however, it suffices to restrict the $\mu$-calculus in order to gain tractable model checking procedures. The alternation free fragment, denoted by $L_{\mu}^{1}$, prohibits the nesting of minimal and maximal fixpoint operators. It allows the formulation of many safety as well as liveness properties. While this fragment is already

T. Margaria and W. Yi (Eds.): TACAS 2001, LNCS 2031, pp. 543-558 2001

(C) Springer-Verlag Berlin Heidelberg 2001 
important on its own, it subsumes the logic CTL 6 which is employed in many practical verification tools. It can be shown that the model checking problem for this fragment is linear in the length of the formula as well as the size of the underlying transition system, and, starting with 4 , several sequential model checking procedures are given in the literature (see 13 for a comparison). The algorithms can be classified into global and local algorithms. Global algorithms require the underlying transition system to be completely constructed while local algorithms compute the necessary part of a transition system on-the-fly. In plain words, global algorithms typically compute the fixpoints in an inductive manner while the local algorithms decide the problem by a depth-first-search. 13 compares the algorithms in detail.

Before starting to think about a concrete algorithm, we should consider its limitations, i.e. its complexity. In complexity theory, it is a well-accepted view that problems within the class NC admit a promising parallel computing algorithm. NC is based on the Boolean circuit model for computation and describes the problems computable in polylogarithmic time with polynomially many processors. It can be shown that $\mathrm{NC}$ is contained in P. Problems outside of $\mathrm{NC}$ are consequently considered to be inherently sequential. However, it is not known whether $\mathrm{NC}=\mathrm{P}$. If not, then especially $\mathrm{P}-$-complete problems cannot be in $\mathrm{NC}$. Hence, we call $\mathrm{P}$-complete problems inherently sequential 8 .

We show that model checking $L_{\mu}^{1}$ is inherently sequential, limiting our enthusiasm for finding a (theoretically) good parallel model checking algorithm. Even worse, depth-first-search is also $\mathrm{P}$-complete, hence, promising parallel local algorithms are unlikely to exist 21 . Despite these theoretical limitations, we present a parallel global model checking algorithm. We implemented it within our verification tool TRUTH 14 and found out that it behaves very well for many practical problems.

Our algorithm is based on a characterisation of the model checking problem for this fragment in terms of two-person games due to Stirling 24 . Strictly speaking, we present a parallel algorithm for colouring so-called game graphs corresponding to the underlying model checking problem. This colouring answers the model checking problem and allows a derivation of a winning strategy. The latter may be employed by the user of a verification tool for debugging the underlying system interactively $\boldsymbol{\bullet} 4$.

Another characterisation of this model checking problem can be given in terms of so-called 1-simple-weak-alternating-Büchi automata $\Gamma$. However, these correspond to game-based model checking 16 . Hence, our algorithm can also be used for checking the emptiness of these automata in parallel.

Until today, not much effort has been taken to consider parallel model checking algorithms. 251 I present parallelised data structures which employ further computers within a network as a substitute for external storage. The algorithms described in $19 \%$ divide the underlying problem into several tasks. However, they are designed in the way that only a single computer can be employed to sequentially handle one task at a time. Stern and Dill 23 show how to carry out a parallel reachability analysis. The distribution of the underlying struc- 
ture is similar to the one presented here. But their algorithm is not suitable for model checking temporal logic formulae. 9 presents a parallel reachability analysis algorithm for BDDs. They argue that many safety properties can be formulated as a reachability problem. In this way, their algorithm allows checking safety formulae. However, liveness properties which can be expressed within $L_{\mu}^{1}$ are not supported. Furthermore, 10 argues that explicit state representation as well as BDDs have application domains in which they outperform the other one. Moreover, BDD-based algorithms generally do not provide counter examples, which are important in practice. Our main contribution is the first parallel model checking algorithm for $L_{\mu}^{1}$ that supports interactive debugging.

The syntax and semantics of the $\mu$-calculus are introduced in the next section. Furthermore, it is shown that $L_{\mu}^{1}$ is inherently sequential. In Section $\mathbf{B}$ we describe model checking games for the $\mu$-calculus and provide an important characterisation of the game graph which will be the basis for our parallel algorithm. Section 4 discusses our parallel model checking procedure and is followed by experimental results. We conclude by summing up our approach as well as giving directions for future work.

\section{The $\mu$-Calculus}

In this section, we recall the syntax and semantics of the (modal) $\mu$-calculus and its alternation free fragment. Furthermore, we show that model checking this fragment is inherently sequential.

\subsection{Syntax and Semantics}

Let $\operatorname{Var}$ be a set of variables and $\mathcal{A}$ a set of actions. Formulae of the modal $\mu$-calculus over $\operatorname{Var}$ and $\mathcal{A}$ in positive form as introduced by $\Pi 1$ are defined as

$$
\varphi::=\text { false } \mid \text { true }|X| \varphi_{1} \wedge \varphi_{2}\left|\varphi_{1} \vee \varphi_{2}\right|[K] \varphi|\langle K\rangle \varphi| \nu X . \varphi \mid \mu X . \varphi
$$

where $X \in \operatorname{Var}$ and $K$ ranges over subsets of actions $\mathcal{A}$. Like 24 , we allow sets of actions instead of single actions appearing in modalities $\|$ It is a simple exercise to extend our approach towards the propositional $\mu$-calculus (cf. Section 4 .

A formula $\varphi$ is normal if every occurrence of a binder $\mu X$ or $\nu X$ in $\varphi$ binds a distinct variable, and no free variable $X$ in $\varphi$ is also used in a binder $\mu X$ or $\nu X$. Every formula can easily be converted into an equivalent normal formula. If a formula $\varphi$ is normal, every bound variable $X$ of $\varphi$ identifies a unique subformula $\mu X . \psi$ or $\nu X . \psi$ of $\varphi$ where $X$ is a free variable of $\psi$.

Let $\mathcal{T}=\left(S, T, \mathcal{A}, s_{0}\right)$ be a labelled transition system where $S$ is a finite set of states, $\mathcal{A}$ a set of actions, and $T \subseteq S \times \mathcal{A} \times S$ denotes the transitions. As usual, we write $s \stackrel{a}{\rightarrow} t$ instead of $(s, a, \bar{t}) \in T$. Furthermore, let $s_{0} \in S$ be the initial state of the transition system. We employ valuations $V$ mapping a variable $X$ to a set of states $V(X) \subseteq S$. Let $V[X / E], E \subseteq S$, be the valuation which is the same as $V$ except for $X$ where $V(X)=E$. Given a labelled transition system

\footnotetext{
${ }^{1}\langle-\rangle_{\varphi}$ is an abbreviation for $\langle\mathcal{A}\rangle_{\varphi}$.
} 
$\mathcal{T}=\left(S, T, \mathcal{A}, s_{0}\right)$ and a formula $\varphi$ over $\operatorname{Var}$ and $\mathcal{A}$, the satisfaction of $\varphi$ wrt. $\mathcal{T}$ and a state $s \in S$ is defined for true, false, disjunction, and conjunction in the usual way, and for the temporal and fixpoint operators as follows:

$$
\begin{aligned}
& \mathcal{T}, s \models_{V}[K] \varphi \text { iff } \forall a \in K: \text { if } s \stackrel{a}{\rightarrow} t \text { then } \mathcal{T}, t \models_{V} \varphi \\
& \mathcal{T}, s \models_{V}\langle K\rangle \varphi \text { iff } \exists a \in K: s \stackrel{a}{\rightarrow} t \text { and } \mathcal{T}, t \models_{V} \varphi \\
& \mathcal{T}, s \models_{V} \mu X . \varphi \text { iff } \forall E \subseteq S: \text { if } s \notin E \text { then } \exists t \in S: t \notin E \text { and } \mathcal{T}, t \models_{V[X / E]} \varphi \\
& \mathcal{T}, s \models_{V} \nu X . \varphi \text { iff } \exists E \subseteq S, s \in E: \text { and } \forall t \in E: \mathcal{T}, t \models_{V[X / E]}^{\varphi}
\end{aligned}
$$

We shorten $\mathcal{T}, s \models_{V} \varphi$ by $\mathcal{T}, s \models \varphi$ for a formula $\varphi$ without any free variables and $\mathcal{T}, s_{0} \models_{V} \varphi$ by $\mathcal{T} \models_{V} \varphi$ and use identifiers like $\varphi, \psi, \ldots$ for formulae, $s, t, \ldots$ for states, and $a, b, \ldots$ for actions of the transition system under consideration. $K$ denotes a set of actions. Whenever the sort of the fixpoint does not matter, we use $\sigma$ for either $\mu$ or $\nu$. For a formula of the $\mu$-calculus, we introduce the notion of subformulae and free and bound variables as usual.

The alternation free fragment of the $\mu$-calculus is that sublogic of the $\mu^{-}$ calculus where no subformula $\psi$ of a formula $\varphi$ contains both a free variable $X$ bound by a $\mu X$ in $\varphi$ as well as a free variable $Y$ bound by a $\nu Y$ in $\varphi$.

Given a labelled transition system $\mathcal{T}$ and a formula $\varphi$, model checking is the problem whether $\mathcal{T}$ satisfies $\varphi$, i.e. whether $\mathcal{T} \models \varphi$. The combined complexity of the model checking problem is its complexity wrt. the product of the size of the transition system and the size of the formula. Its program complexity considers the complexity only wrt. the size of the transition system.

In 26 , it was shown that the combined complexity for the alternation free $\mu$-calculus is $\mathrm{P}$-complete and, for a version of the alternation free $\mu$-calculus employing two actions, that its program complexity is $\mathrm{P}$-complete. $\mathrm{I} 2$ shows the latter result by using a formula with two propositions. We strengthen both results by employing neither propositions nor any action labelling.

Lemma 1. The program complexity of the alternation free $\mu$-calculus is $P$-hard.

Proof. We reduce the $\mathrm{P}-$ complete Game Problem $\mathrm{x}$ to checking a formula of the alternation free $\mu$-calculus wrt. a corresponding labelled transition system. A two player game is a tuple $G=\left(P_{1}, P_{2}, M, W_{0}, s\right) . P_{1}$ and $P_{2}, P_{1} \cap P_{2}=\emptyset$, are positions, in which it is the turn of Player 1 or Player 2, resp. $M \subseteq\left(P_{1} \times\right.$ $\left.P_{2}\right) \cup\left(P_{2} \times P_{1}\right)$ is the set of moves the respective player can make. $W_{0} \subseteq P_{1} \cup P_{2}$ denotes the succeeding positions, $s \in P_{1}$ the starting position. The players move alternately beginning with Player 1 . We call $x \in P_{1} \cup P_{2}$ winning iff either $x$ is succeeding $\left(x \in W_{0}\right)$, or $x \in P_{1}$ and there is a winning $y \in P_{2}$ such that $(x, y) \in M$, or $x \in P_{2}$ and for all $(x, y) \in M, y$ is winning. The Game Problem is the question whether $s$ is winning.

Corresponding to this, we define a transition system $\mathcal{T}_{G}=\left(P_{1} \cup P_{2}, T\right)$ by $T=\left(M-\left\{(p, q) \in M \mid p \in W_{0}\right\}\right) \cup\left\{(p, p) \in P_{1} \times P_{1} \mid p \notin W_{0}\right.$ and there is no transition from $p$ in $M\}$.

$T$ is defined in the way that every deadlock state, i.e. state with no outgoing edges, is a state of $W_{0}$ or a state of $P_{2}$ in which Player 2 is not able to move. Hence, deadlocks are winning. They can be characterised in the $\mu$-calculus by $\varphi_{W_{0}}=[-]$ false where a formula $[-] \varphi$ indicates that $\varphi$ is satisfied in all successor 
states. Further winning positions for Player 1 are states of $P_{1}$ such that there is a successor state (in $P_{2}$ ) whose direct successors (in $P_{1}$ ) are all winning. Hence, the formula $\varphi=\mu X .\left(\langle-\rangle[-] X \vee \varphi_{W_{0}}\right)$ is satisfied in exactly those positions of $P_{1}$ which are winning where $\langle-\rangle \varphi$ guarantees the existence of a successor state in which $\varphi$ holds. Note that $\varphi$ may be satisfied in further positions of $P_{2}$ which does not bother us. We conclude that $s$ is winning in the game $G=\left(P_{1}, P_{2}, M, W_{0}, s\right)$ iff $\mathcal{T}_{G}, s \models \varphi$. Observe that the construction of the transition system can be done in LOGSPACE. Note, we do not make use of propositions. Furthermore, we manage without actions at all by slightly adapting the modal fragment of our logic.

Together with a lineartime algorithm 12 13, we have the following theorem:

Theorem 1. Model checking the alternation free $\mu$-calculus is inherently sequential wrt. the combined complexity as well as the program complexity.

\section{Games for the $\mu-$ Calculus}

Given a labelled transition system $\mathcal{T}=\left(S, T, \mathcal{A}, s_{0}\right)$ and a formula $\varphi$ over $\operatorname{Var}$ and $\mathcal{A}$, we are able to define the model checking game. Its board is the Cartesian product $S \times S u b(\varphi)$ of the set of states and the set of subformulae. The game is played by two players, namely $\forall$ belard (the pessimist), who wants to show that $\mathcal{T}, s_{0} \models \varphi$ does not hold, whereas $\exists$ loise (the optimist) wants to show the opposite.

The model checking game $G(s, \varphi)$ is given by all its plays, i.e. (possibly infinite) sequences $C_{0} \rightarrow_{p_{0}} C_{1} \rightarrow_{p_{1}} C_{2} \rightarrow_{p_{2}} \ldots$ of configurations, where $C_{0}=$ $(s, \varphi)$ and for all $i, C_{i} \in S \times S u b(\varphi)$ and $p_{i}$ is either $\exists$ loise or $\forall$ belard. We write $\rightarrow$ instead of $\rightarrow_{p_{i}}$ if we abstract from the player. The players do not have to move alternately, instead, the next turn is determined by the current subformula of $\varphi$. Hence, the second component of a configuration $C_{i}$ determines the player $p_{i}$ who is to choose the next move. $\forall$ belard makes universal $\rightarrow \forall$-moves, $\exists$ loise makes existential $\rightarrow_{\exists}$-moves. More precisely, whenever

1. $C_{i}=(s, \mathrm{false})$, then the play is finished.

2. $C_{i}=\left(s, \psi_{1} \wedge \psi_{2}\right)$, then $\forall$ belard chooses $\varphi=\psi_{1}$ or $\varphi=\psi_{2}$, and $C_{i+1}=(s, \varphi)$.

3. $C_{i}=(s,[K] \psi)$, then $\forall$ belard chooses $s \stackrel{a}{\rightarrow} t$ with $a \in K$ and $C_{i+1}=(t, \psi)$.

4. $C_{i}=(s, \nu X . \psi)$, then $C_{i+1}=(s, \psi)$.

5. $C_{i}=(s$, true $)$, then the play is finished.

6. $C_{i}=\left(s, \psi_{1} \vee \psi_{2}\right)$, then $\exists$ loise chooses $\varphi=\psi_{1}$ or $\varphi=\psi_{2}$, and $C_{i+1}=(s, \varphi)$.

7. $C_{i}=(s,\langle K\rangle \psi)$, then $\exists$ loise chooses $s \stackrel{a}{\rightarrow} t$ with $a \in K$ and $C_{i+1}=(t, \psi)$.

8. $C_{i}=(s, \mu X . \psi)$, then $C_{i+1}=(s, \psi)$.

9. $C_{i}=(s, X)$ and $X$ identifies $\varphi$, then $C_{i+1}=(s, \varphi)$.

As the moves $1,4,5,8$ and 9 are deterministic, no player needs to be charged with them. With regard to the winning strategies and the algorithm, we will speak of $\forall$ belard-moves in cases $1-4$ and 9 if $\sigma=\mu$, and $\exists$ loise-moves in all other cases. $C_{i}$ is called $\forall$-configuration or $\exists$-configuration, respectively.

$\forall$ belard wins a play $G$, iff 
- $G=C_{0} \rightarrow \cdots \rightarrow C_{n}$ and $C_{n}=(s$, false $)$ for any state $s$.

$-G=C_{0} \rightarrow \cdots \rightarrow C_{n}$ and $C_{n}=(s,\langle K\rangle \varphi)$ and $\nexists t: s \stackrel{a}{\rightarrow} t$ for any $a \in K$.

- $G=C_{0} \rightarrow \cdots$ has infinite length and the outermost fixpoint which is unwinded infinitely often is a $\mu$-fixpoint.

Dually, $\exists$ loise wins a play $G$, iff a configuration with the formula true is reached, $\forall$ belard gets "stuck", or the outermost fixpoint which is unwinded infinitely often is a $\nu$-fixpoint 24 .

Please note that, given a transition system and a formula, there are several plays and these not necessarily have the same winner.

To characterise plays, we introduce the notion of witnesses and judgements. Configurations $C$ in which no move is possible are called judgements. A judgement is further called $\exists$-judgement $(\forall$-judgement) iff it is an $\exists$-configuration $(\forall$-configuration). For finite plays, it is obvious that $\exists$ loise ( $\forall$ belard) wins the play iff it contains an $\exists$-judgement ( $\forall$-judgement).

Configurations of the form $(s, X)$ where $s$ is any state of the transition system and $X$ is a variable are called witnesses since in the following move, $X$ is unwinded. If $X$ is bound by a $\mu$-quantifier, it is called an $\forall$-witness, otherwise $\exists$-witness. Witnesses have a natural partial order given by the nesting within the originating formula. A witness $(s, X)$ is less than $(s, Y)$ iff $\sigma X . \varphi(X, Y)$ is a subformula of $\sigma^{\prime} Y . \psi(X, Y)$ where $\eta(X, Y)$ means that $\eta$ may contain the free variables $X, Y$. For infinite plays, it is easy to see that it has a unique maximal witness and that the winning condition can be formulated as $\forall$ belard wins iff this witness is an $\forall$-witness, $\exists$ loise wins iff it is an $\exists$-witness. Please note that no configuration can be a judgement as well as a witness.

A strategy is a set of rules for a Player $p$ telling her or him how to move in the current configuration. A winning strategy now guarantees that the play which $p$ plays regarding the rules will be won by $p$. 94 shows that the model checking problem for the $\mu$-calculus is equivalent to finding a winning strategy for one of the players: Let $\mathcal{T}$ be a transition system with starting state $s$, and let $\varphi$ be a $\mu$-calculus formula. $\mathcal{T}, s \models \varphi$ implies that $\exists$ loise has a winning strategy starting at $(s, \varphi)$, and $\mathcal{T}, s \forall \forall$ implies that $\forall$ belard has a winning strategy starting at $(s, \varphi)$. Since a formula either holds or is falsified, this result also implies that model checking games are determined, i.e., for every game, either $\forall$ belard or $\exists$ loise has a winning strategy.

All possible plays for a transition system $\mathcal{T}$ and a formula $\varphi$ are captured in the game graph whose nodes are the elements of the game board (the possible configurations) and whose edges are the players' possible moves. The game graph can be understood as an and-/or-graph where the or-nodes (denoted by $\bigvee)$ are $\exists$-configurations and and-nodes (denoted by $\Lambda$ ) are $\forall$-configurations. Furthermore, the notion of witnesses and judgements carries over without any modification. A play corresponds to a path in the game graph and vice versa.

In the following, we concentrate on the alternation free $\mu$-calculus. The following characterisation of the game graph for this fragment is useful for formulating a sequential algorithm and essential for our parallel algorithm. 
Theorem 2. Let $\mathcal{T}$ be a labelled transition system and $\varphi$ a formula of the alternation free $\mu$-calculus. Furthermore, let $(Q, E)$ be their game graph. Then there exists a collection of $Q_{1}, \ldots, Q_{m}$ such that the following holds:

1. the collection of the $Q_{i}$ is a partition of $Q$, i.e., $Q=\bigcup_{i \in\{1, \ldots, m\}} Q_{i}$ and for all $i, j \in\{1, \ldots, m\}$ with $i \neq j$, it holds $Q_{i} \cap Q_{j}=\emptyset$.

2. The subgraph induced by $Q_{i}$ is exactly one of

(a) a non-trivial maximal strongly connected component (Type I) $\mathbf{2}$

(b) a singleton containing a judgement (Type II).

(c) a maximal directed acyclic graph without any judgements (Type III).

3. every $Q_{i}$ of Type I either contains at least one $\exists$-witness and no $\forall$-witnesses or contains at least one $\forall$-witness and no $\exists$-witnesses.

4. there is a partial order $\leq$ on the $Q_{i}$ 's such that for every $q \in Q_{i}$ and $q^{\prime} \in Q_{j}$ with an edge from $q$ to $\bar{q}^{\prime}$, we have $Q_{j} \leq Q_{i}$. Thus, moves from a configuration in $Q_{i}$ lead to configurations in either the same $Q_{i}$ or a lower $Q_{j}$.

Proof. The proof is inspired by a characterisation of the game graph in terms of weak alternating automata 16 . First, consider the nodes of maximal non-trivial strongly connected components. These only occur because of unwinding fixpoint formulae. Hence, they contain witnesses. Alternation freeness now guarantees that these are all of the same kind, i.e. either $\exists$-witnesses or $\forall$-witnesses. Second, consider the leaves of the game graph, i.e. configurations without outgoing edges. These cannot be members of strongly connected components of Type I. By definition, every such $Q_{i}$ is a judgement. Third, it is now easy to see that all remaining nodes belong to directed acyclic graphs which do not contain any leaves of the original game graph. Please note that maximality of the strongly connected components guarantees the order defined to be a partial order.

To prove our parallel algorithm to be free from deadlock, we need the following insight which holds since on every cycle, a fixpoint formula is unwinded.

Proposition 1. Every strongly connected component of a game graph with more than one element contains at least one witness.

Let us sketch a sequential algorithm deciding which player has a winning strategy 5 . It labels a configuration $q$ by green or red, depending on whether ᄏloise or $\forall$ belard has a winning strategy for the game starting in this configuration $q$. It will be extended to a parallel version in the next section.

Let us consider a game graph. By Theorem $\boldsymbol{\nu}$ there exists a partition of its nodes $Q$ into disjoint $Q_{i}$ of Type I-III, and every $Q_{i}$ of Type I either contains $\exists$-witnesses or $\forall$-witnesses. Also, there exists a partial order $\leq$ on the $Q_{i}$ such that for $q \in Q_{i}$ and $q^{\prime} \in Q_{j}$ for which there is a possible move from $q$ to $q^{\prime}$, we have $Q_{j} \leq Q_{i}$. As seen before, every infinite play gets trapped within a single $Q_{i}$, and the winner depends on whether $Q_{i}$ contains $\exists$-witnesses or $\forall$-witnesses. By Prop. II every infinite play visits such a witness infinitely often.

The game graph can be coloured by processing all $Q_{i}$ upwards according to the partial order. To make the algorithm deterministic, enlarge the partial order

${ }^{2}$ A component is called non-trivial if it contains a least two nodes. 
on the $Q_{i}$ to a total order. Let $Q_{i}$ be minimal wrt. $\leq$. Then it is of Type I or Type II. Furthermore, from any configuration of $Q_{i}$, every move leads to $Q_{i}$. If in $Q_{i}$ there is an $\exists$-witness or $Q_{i}$ consists of an $\exists$-judgement, all its nodes are labelled with green, otherwise red.

Once a configuration $q \in Q_{i}$ is labelled with red or green, its predecessors are labelled if possible. That means, an $\bigwedge$-node is labelled with red if $q$ is $r e d$, but labelled green, if all successors are green. An $\bigvee$-node is treated dually. Furthermore, if a node could be labelled, its outgoing edges are erased. Such labelling is propagated further.

Let $Q_{j}$ be the next set of configurations wrt. the total order. Then, all configurations in $Q_{i} \leq Q_{j}$ are already coloured by either red or green. If $Q_{j}$ is of Type III, all of its configurations must be labelled due to the propagation described before. For a set of Type I, some unlabelled configurations might remain. These are labelled according to the type of witnesses in $Q_{j}$, i.e. with red if $Q_{j}$ has $\forall$-witnesses, otherwise with green.

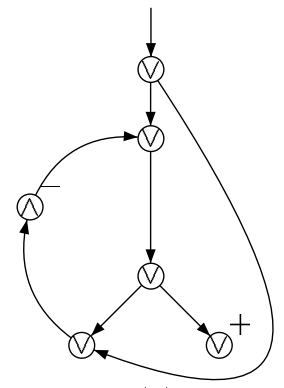

(a)

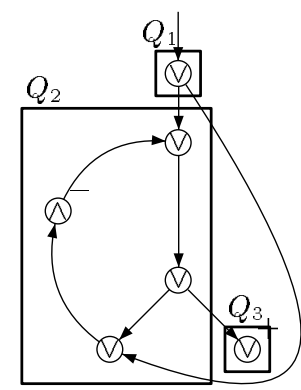

(b)

Fig. 1. State graph (a) and its partition (b)

Let us consider Figure II Part (b) is a partition of the game graph shown in (a). It contains components of types I, II, and III. $Q_{1}$ is a directed acyclic graph, $Q_{2}$ a non-trivial maximal strongly connected component, and $Q_{3}$ is a singleton containing a $\exists$-judgement (denoted by ${ }^{+}$). The components can be ordered like $Q_{3}<Q_{2}<Q_{1}$. Since the minimal $Q_{3}$ contains an $\exists$-judgement, it will be labelled with green. The $V$-node from $Q_{2}$ to $Q_{3}$ and subsequently all nodes will therefore be labelled with green as well. Note how the $\forall$-witness (denoted by ${ }^{-}$) has no influence here, since all nodes are coloured already.

\section{Parallel Model Checking}

Given a transition system and an $L_{\mu}^{1}$-formula, our approach is both to construct the game graph as well as to determine the colour of its nodes in parallel.

\subsection{Distributing the Game Graph}

It is obvious that the construction of the game graph can be carried out in parallel by a typical breadth-first strategy. Given a node $q$, determine its successors $q_{1}, \ldots, q_{n}$. Now, the successors can be processed in the same manner in parallel. 
However, to obtain a terminating procedure, only exactly the $q_{i}$ not processed before must be expanded. All states generated have to be stored within the NOW, and load sharing must be guaranteed. On a shared memory architecture, this does not involve big conceptual problems. For distributed memory machines, however, this is a little bit more difficult.

A first idea might be to distribute the first $q_{1}, \ldots, q_{n}$ to the first $n$ processors, and these process the $q_{i}$ as described before and distribute the successors to the next processors. However, deciding whether a $q_{i}$ was processed before becomes an expensive operation. Every processor could have processed $q_{i}$ and should therefore be consulted. In the worst case, for every node, such a broadcast is required. This yields no reasonable algorithm.

A different, often-employed way to store graphs on a distributed memory machine is to divide the graph's adjacency matrix $M \in\{0,1\}^{|V| \times|V|}$ into equal sized blocks and to store each block on a single processor 22 . This has several advantages. First, the blocks of the matrix can be generated in parallel. Second, given nodes $p, q \in V$, it is easy to check whether there is an edge from $p$ to $q$, i.e. whether $M_{p, q}=1$. Since there is a unique location for the block of the matrix containing the value for the pair $(p, q)$, a single communication is needed. Third, every processor gets the same amount of data.

For our problem, this approach cannot be used. The number of nodes of our graph is unknown a priori but computed while constructing the graph $\mathbf{1}$ Hence, the partition of the game graph into blocks cannot be determined in advance.

We propose the following way to construct and store the graph which is inspired by the work pool presentation of 15 and is similarly applied in $\% 3$. Let $f$ be a function mapping the states of the game graph to a processor of our network. Usually, one takes a function in the spirit of a hash function assigning to every state an integer and subsequently its value modulo the number of processors. Then, $f$ determines the location of every state within the network uniquely and without global knowledge. In a breadth-first manner, starting with the initial state $q_{0}$ of the game graph, the state space can be constructed in parallel with the help of $f$ in the following way. Given a state $q$ (and possibly some of its direct predecessors), send it to its processor $f(q)$. If $q$ is already in the local store of $f(q)$, then $q$ is reached a second time, hence the procedure stops. If predecessors of $q$ were sent together with $q$, the list of predecessors is augmented accordingly. If $q$ is not in the local memory of $f(q)$, it is stored there together with the given predecessors as well as all its successors $q_{1}, \ldots, q_{k}$, the states within the formula $\delta(q, a)$ which are computed. These are sent in the same manner to their (wrt. $f$ ) processors, together with the information that $q$ is a direct predecessor. The corresponding processes update their local memory similarly.

\subsection{Labelling the Game Graph}

Given the game graph, a first possibility for labelling the nodes with red and green would be to apply a depth-first-search as done by sequential algorithms.

\footnotetext{
${ }^{3}$ In the context of model checking, the transition system is not given explicitly but expanded at run-time from a formal system description.
} 
However, since this problem is $\mathrm{P}$-complete 21 , there is no hope to get a suitable parallel algorithm by adapting the ideas of depth-first-search, and there is no reason to do this.

Another possibility for labelling the nodes with red and green is applying typical algorithms for generating strongly connected components of our game graph in parallel which will be labelled in a second step 22 . However, labelling a connected component requires the knowledge whether the component can be "successfully abandoned" (cf. Section 3. It is neither clear how to obtain this information from the graph computed by these algorithms nor to modify these algorithms in the way that this information can be gathered easily.

We therefore propose the following method. The parallel colouring process is carried out speculative. For example, given an $\exists$-witness $q$, it determines an accepting component. This node is coloured green unless there is an $\bigwedge$-node with an edge to a lower component which is coloured red. The parallel algorithm, however, labels the witness $q$ with green. Furthermore, a notification is sent to its direct predecessors $q_{1}, \ldots, q_{k}$. This notification tells each $q_{i}$ that $q$ changed its colour. Hence, they recompute their own colour according to the following obvious rule: If $q_{i}$ is an $\bigvee$-node, then it is labelled with green if one of its successors is green. If all successors are red, it is labelled with red. Otherwise, some successors have no label yet and no colour is assigned to the current node. For an $\bigwedge$-node, the dual is carried out. If the colour of $q_{i}$ has changed, it sends a notification to its predecessors where the same procedure starts again. Otherwise, the procedure is done. It is clear that the predecessors can be processed in parallel. The whole algorithm stops if all notifications are processed.

Theorem 3. The algorithm described before labels a node $(s, \psi)$ of the game graph with green if $\mathcal{T}, s \models \psi$. Otherwise, the node is labelled with red.

Proof. We give a sketch of the proof. The termination of the algorithm can easily be seen by recalling that the game graph can be divided into components as aforementioned which are partially ordered. Labelling notifications are sent either within the current component or propagated to a higher one (wrt. the partial order). Since the colour operation is monotone (in the obvious sense), only a finite number of labelling notifications is generated.

When the algorithm terminates, the game graph is entirely labelled (completeness), because the components are either leaves (in which case they are labelled as described before), they contain a witness (cf. Proposition $\mathbf{\|}$, in which case the speculative part of the algorithm jumps in and starts the labelling of this component, or completely depend on a lower component.

The sequential algorithm labels the nodes with a correct colour by processing the mentioned components according to the partial order. The labelling remains correct if the labelling of higher (wrt. the partial order) components is done speculative and corrected as soon as the correct colour of the lower component is determined. Note that the colour of leaves and leaf components (i.e. components which are minimal for the partial order) are correctly labelled from the beginning.

It should be mentioned that for an implementation, the two steps of constructing the game graph and labelling the nodes are carried out concurrently 
(cf. Section 4.3. The combined algorithm stops if no further labelling or state expansion steps have to be processed. For this task, we employ the DFG token termination algorithm, as presented in 5. Due to space constraints, we will not go into the details of this algorithm.

\subsection{The Algorithm}

To describe our approach in more detail, we show an algorithm in pseudo code which combines the task of constructing and labelling the game graph in parallel. The termination check is omitted to simplify the presentation. This algorithm runs on every machine within the NOW. Note that initially one machine has to send the root of the game graph to its processor to start the procedure.

Let us consider Figure Each processor's part of the graph is stored there in a relational structure consisting of one tuple for every node already processed of the form (node, colour, preds, succs) where node is the node together with its colour, its predecessors preds, and its successors together with their colours (succs, line 3). We introduce the colour white to denote unlabelled nodes. In line 7 , a message is received. If the message requests to expand a given node, it is checked whether this node has already been processed before (line 10). If so, it might have a colour (not equal to white) which will be propagated to the new predecessors (lines 12-13). In any case, new predecessors are stored (line 11). If the delivered node has not been processed before (line 14), its successors are computed (with an initially white colour) and propagated to the corresponding processors (lines 15-18). Furthermore, it must be checked whether a labelling process must be initiated, i.e. whether the current node fulfils the requirements for being either an $\exists$ - or an $\forall$-witness. If so, all predecessors are informed about the current node's colour (lines 19-25).

The second type of messages which are received are colouring messages (line 26) informing that a node's child has changed its colour. The current settings for node are extracted (line 27), and the new colour of the corresponding child is stored (line 28). If now the evaluation of the node's colour according to Section 4.2 yields a different colour than the old one, then all predecessors are informed in the previous way (lines 29-33).

It is easy to see that the space required by our algorithm is linear in the size of the game graph. The worst case run-time, however, is a factorial of its size. This case might turn up when the components of the game graph are of Type II, linearly ordered and alternating, i.e. $Q_{i}$ contains an $\exists$-witness and $Q_{i+1}$ contains an $\forall$-witness. Now, in every component a (speculative) recolouring up to the maximal (root) component may be initiated.

Since we already observed that the model checking problem for the considered fragment of the $\mu$-calculus is unlikely to be in NC, we were warned that a parallel algorithm might not be optimal in every case. Despite these theoretical limitations, in practice the behaviour turns out to be feasible (cf. Section $\mathbf{n}$. An explanation for this fact is that the aforementioned kinds of game graphs require formulae with deeply nested fixpoints, which rarely occur as typical specifications. 
5

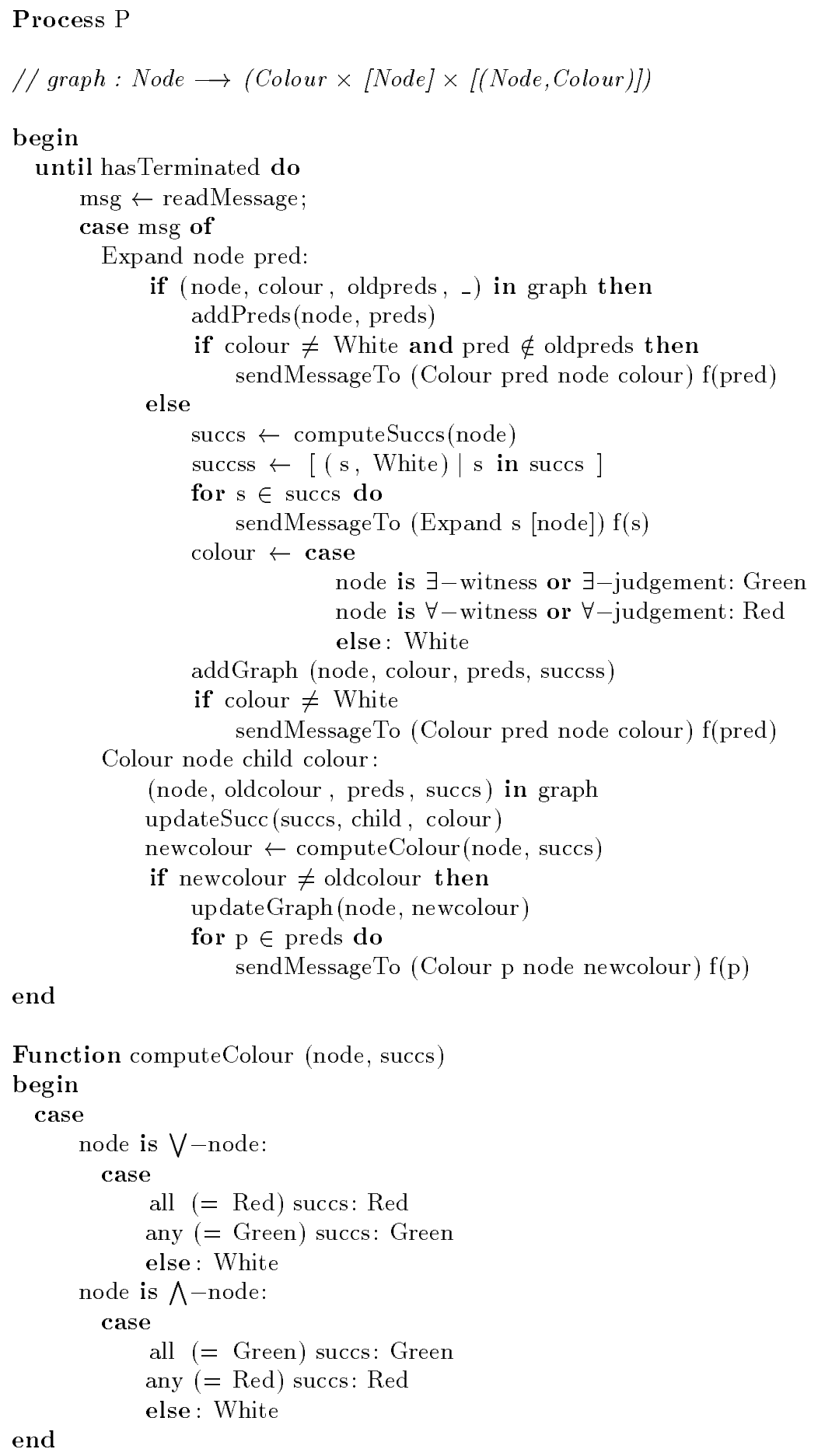

Fig. 2. A parallel construction and labelling algorithm 
Let us consider the graph in Figure $\mathbf{3}$ as a simple example. It suggests that the distribution function $f$ will map the given nodes onto two processors as shown. Starting with node 1, its successors $(2,5)$ are computed and sent to processors $p_{1}$ and $p_{2}$, resp. Now, 2 and 5 can be expanded in parallel with the effect that nodes 3 and 6 are sent to $p_{2}$. The successors of 3 are 5 and 4 which are delivered to $p_{2}$. Since 6 is an $\forall$-witness (indicated by ${ }^{-}$), it is labelled with red, initiates a relabelling of 5 , and issues an expand-2-message to $p_{1}$, which notices that

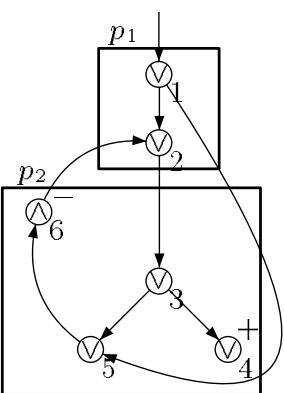

Fig. 3. A partition 2 is already expanded and registers 6 as one of 2's predecessors. $p_{2}$ carries on with expanding 4 , noticing that it is an $\exists$-judgement (indicated by ${ }^{+}$), and sends this information to 3 . Next, the red-labelling of 5 is propagated to 3 and 1 . However, 3 can now determine green as its colour and sends it to its predecessor. 2 propagates green to 1 and 6 . Finally, the whole graph is labelled green.

\section{$5 \quad$ Experimental Results}

We have tested our approach within our verification platform TruTH 14. We implemented the distribution routine on its own as well as the combined labelling routine described in the previous section. As implementation language we have chosen the purely functional programming language Haskel $\mathbf{A}$, which enabled us to embed this algorithm in the infrastructure of our verification tool TRUTH and also to prototype a concise reference implementation. The actual Haskell source code of the algorithm has less than 300 lines of code. The communication layer of our implementation is based upon MPICH $\mathbf{f}$, an implementation of the MPI (Message Passing Interface) standard.

Now we will show some results we achieved when verifying properties of certain system specifications. Figures 4 and $\boldsymbol{b}$ show the measured results of state distribution and the speedup when running our implementation on a NOW consisting of up to 52 processors and a total of $13 \mathrm{~GB}$ main memory. They are connected with a conventional 100MBit Fast-Ethernet network.

The distribution routine shows that our approach is very well suited for constructing large game graphs. We were able to construct graphs with several hundred thousands of states within minutes. The game graph of the largest example we have constructed so far, a quad-parallel instance of the Alternating Bit Protocol 18, consists of more than 1.6 million states, and we get a homogeneous distribution of the state space on the workstations (Figure 4. The distribution depends on our hash function $f$, and the results are quite good compared to its simplicity. In fact, all our measurements showed similar results, provided the size of the graph is reasonably larger than the number of used workstations.

Our approach also scales very well with regard to the overall runtime (Figure 5 . Unfortunately, because of the size of the game graphs we inspected, we

\footnotetext{
4 http://haskell.org/

5 http://ww-unix.mcs . anl.gov/mpi/mpich/
} 


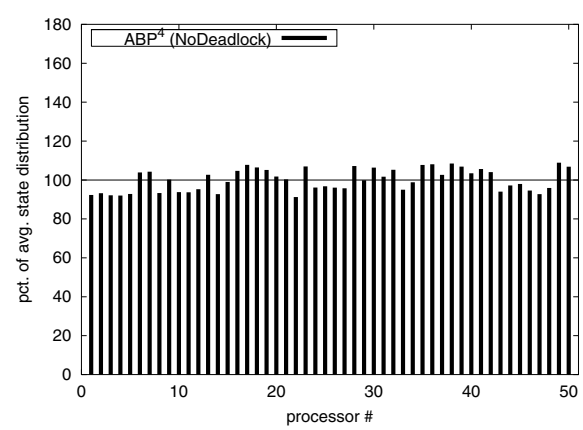

Fig. 4. state distribution

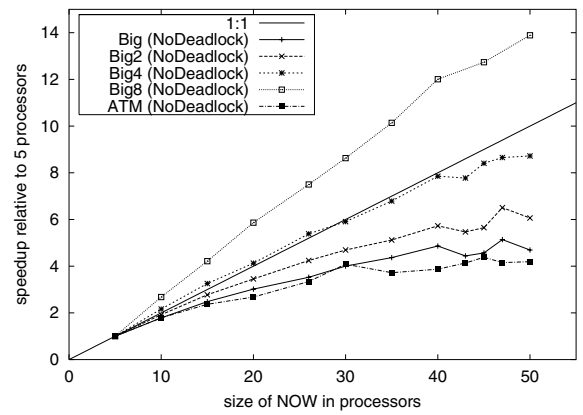

Fig. 5. runtime results

did not get results when running the algorithm on less than five workstations due to memory restrictions. Therefore the shown speedups are calculated relative to 5 processors instead of one. We found that we gain a linear speedup for reasonably large game graphs (in fact, for graphs with more than 500.000 states we even got superlinear speedups, which we will discuss later). The results are especially satisfying, if one considers that - for reasons of simplicity - we did not try to employ well-known optimisation means, for example reducing the communication overhead by packing several states into one message.

Due to our choice of Haskell as implementation language and its inherent inefficiency, we did not focus on optimising the internal data structures either. We use purely functional data structures like balanced trees and lists rather than destructively updateable arrays or hash tables. This is also the reason for the superlinear speedups we remarked before. We found that the overhead for insertions and lookups on our internal data structures dramatically increases with the number of stored states. We verified this by running all processes on a single processor in parallel and replacing the network message passing with inter-process communication. The expected result would have been to find similar runtimes as one process would achieve in this situation, or even slightly worse due to operating system context switches between the processes running in parallel. But we found that there is a significant speedup because the internal data structures are less crowded so that lookups and insertions are considerably cheaper.

Comparing our approach to the implemented sequential game-based depthfirst-search model checking algorithm for $L_{\mu}^{1}$, we have to learn that it is not possible for small examples to beat it. There are two reasons for that. In many cases, a formula can be proven or falsified by considering only a part of the game graph. Even parallel power does not outperform in these cases. Second, the communication between processors is dramatically slower within a NOW compared to accessing memory. Hence, as long as a problem fits into main memory, it is difficult to beat a sequential algorithm by a parallel one running on a NOW.

The situation changes completely when most of a huge game graph has to be checked for proving a formula. This situation arises for example in the frequent case that a NoDeadlock formula is considered. To check whether a system description contains any deadlock requires the whole game graph to be analysed. 
For large systems (several hundred thousand states), the parallel version beats the sequential one. More important, we were able to verify certain systems with the help of our parallel algorithm while the sequential failed due to memory restrictions.

\section{Conclusion}

In this paper, we presented a parallel game-based model checking algorithm for an important fragment of the $\mu$-calculus. The demand for parallel algorithms becomes visible by considering the memory and run-time consumptions of sequential algorithms. Since the employed fragment of the $\mu$-calculus subsumes the well-known logic CTL, it is of high practical interest. We have implemented our approach within the verification platform TRUTH. Systems with a million of states could be constructed within half an hour on a NOW consisting of up to 52 processors. We found out that the algorithm scales very well wrt. run-time and memory consumption when enlarging the NOW. Furthermore, the distribution of states on the processors is homogeneous.

Compared to the also implemented on-the-fly sequential model checking algorithm for $L_{\mu}^{1}$, we learned that for simple examples a parallel global algorithm cannot outperform a local one. This is especially true for formulae like true $\vee \varphi$ being checked, where $\varphi=\nu X . \Phi$ yields a considerably big part of the resulting game graph. A local algorithm would be able to almost instantaneously present a solution, since the formula is dominated by an $\exists$-judgement (true).

To improve our algorithm for such cases, we head towards an "almost local" variant, which not only uses two colours but colour weights, with which e.g. the propagation of safe colours (resulting from minimal components $Q_{i}$ ) can be tracked better. So we eventually short-circuit the colouring process.

However, considering real world specifications yielding millions of states, even the here presented parallel algorithm gains the upperhand. Answers are computed more quickly, and, more important, there are numerous cases in which the sequential algorithm fails because of memory restrictions and the parallel version is able to prove a formula. From the practical point of view, it is a central feature of a verification tool to give an answer in as many cases as possible.

While our approach is already of practical interest since it allows to check larger systems, it should also be considered as a further attempt to develop parallel model checking algorithms. More research should be carried out in this direction. Especially, on-the-fly model checking and partial order reduction 20 should be analysed with respect to parallelisation. Furthermore, different (especially non-P-complete) specification logics might provide better parallel model checking algorithms.

\section{References}

1. S. Basonov. Parallel implementation of BDD on DSM systems. Master's thesis, Computer Science Department, Technion, 1998. 
2. G. Cabodi, P. Camurati, and S. Que. Improved reachability analysis of large FSM. In Proc. IEEE Int. Conf. on Computer Aided Design, pages 354-360. IEEE Computer Society Press, June 1996.

3. E. M. Clarke and J. Wing. Formal methods: State of the art and future directions. Technical Report CMU-CS-96-178, Carnegie Mellon University (CMU), Sept. 1996.

4. R. Cleaveland and B. Steffen. A linear-time model-checking algorithm for the alternation-free modal mu-calculus. In Proc. Computer Aided Verification (CAV '91), volume 575 of $L N C S$, Springer, 1992

5. E. W. Dijkstra, W. H. J. Feijen, and A. J. M. van Gasteren. Derivation of a termination detection algorithm for distributed computations. Information Processing Letters, 16(5):217-219, June 1983.

6. E. A. Emerson and E. M. Clarke. Using branching time temporal logic to synthesize synchronization skeletons. Science of Computer Programming, 2(3):241-266, 1982.

7. S. Gnesi et. al., A formal specification and validation of a critical system in presence of byzantine errors. In Proc. 6th Int. Conf. on Tools and Algorithms for the Construction and Analysis of Systems, number 1785 in LNCS. Springer, 2000.

8. R. Greenlaw, H. J. Hoover, and W. L. Ruzzo. Limits to Parallel Computation: P-Completeness Theory. Oxford University Press, 1995.

9. T. Heyman, D. Geist, O. Grumberg, and A. Schuster. Achieving scalability in parallel reachability analysis of very large circuits. In Computer Aided Verification, 12th Int. Conf., volume 1855 of LNCS, pages 20-35. Springer-Verlag, June 2000.

10. A. J. Hu, G. York, and D. L. Dill. New techniques for efficient verification with implicitly conjoined BDDs. In 31st Design Automation Conference, 1994.

11. D. Kozen. Results on the propositional mu-calculus. Theoretical Computer Science, 27:333-354, Dec. 1983.

12. O. Kupferman, M. Y. Vardi, and P. Wolper. An automata-theoretic approach to branching-time model checking. Journal of the ACM, 47(2):312-360, Mar. 2000.

13. M. Lange. Spielbasiertes Model-Checking für den alternierungsfreien mu-Kalkül. Master's thesis, Aachen, University of Technology, 1999. (German).

14. M. Lange, M. Leucker, T. Noll, and S. Tobies. Truth - a verification platform for concurrent systems. In Tool Support for System Specification, Development, and Verification, Advances in Computing Science. Springer-Verlag, 1999.

15. B. P. Lester. The Art of Parallel Programming. Prentice Hall, 1993.

16. M. Leucker. Model checking games for the alternation free mu-calculus and alternating automata. In Proc. 6th Int. Conf. on Logic for Programming and Automated Reasoning, volume 1705 of $L N A I$, pages 77-91. Springer, 1999.

17. K. L. McMillan. Symbolic Model Checking. Kluwer Academic Publishers, 1993.

18. R. Milner. Communication and Concurrency. Prentice Hall, 1989.

19. A. A. Narayan, J. J. J. Isles, R. K. Brayto, and A. L. Sangiovanni-Vincentelli. Reachability analysis using partitioned-roBBDs. In Proc. IEEE Int. Conf. on Computer Aided Design, pages 388-393. IEEE Computer Society Press, June 1997.

20. D. Peled. Ten years of partial order reduction. In CAV, Computer Aided Verification, number 1427 in LNCS, pages 17-28, Vancouver, BC, Canada, 1998. Springer.

21. J. H. Reif. Depth-first search is inherently sequential. Information Processing Letters, 20(5):229-234, June 1985.

22. S. H. Roosta. Parallel Processing and Parallel Algorithms. Springer, 1999.

23. U. Stern and D. L. Dill. Parallelizing the Mure verifier. In Proc. 9th Int. Conf. Computer Aided Verification, volume 1254 of LNCS, Springer, 1997.

24. C. Stirling. Games for bisimulation and model checking, July 1996. Notes for Mathfit Workshop on finite model theory, University of Wales, Swansea,

25. A. L. Stornetta. Implementation of an efficient parallel BDD package. Master's thesis, University of California, Santa Barbara, 1995.

26. S. Zhang, O. Sokolsky, and S. A. Smolka. On the parallel complexity of model checking in the modal mu-calculus. In Proc. 9th Annual IEEE Symposium on Logic in Computer Science, pages 154-163, 1994. IEEE Computer Society Press. 